POS $\quad$ PROCEEDINGS

\title{
Overlap Valence Quarks on a Twisted Mass Sea
}

\section{K. Cichy*i}

Adam Mickiewicz University, Faculty of Physics, Umultowska 85, 61-614 Poznań, Poland

E-mail: kcichy@amu.edu.pl

V. Drach, E. Garcia-Ramos, G. Herdoiza, K. Jansen

NIC, DESY, Platanenallee 6, D-15738 Zeuthen, Germany

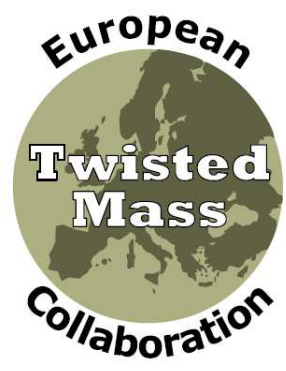

We present the results of an investigation of a mixed action approach of overlap valence and maximally twisted mass sea quarks. Employing a particular matching condition on the pion mass, we analyze the continuum limit scaling of the pion decay constant and the role of chiral zero modes of the overlap operator in this process. We employ gauge field configurations generated by the European Twisted Mass Collaboration with linear lattice size $L$ ranging from 1.3 to $1.9 \mathrm{fm}$. The continuum limit is taken at a fixed value of $L=1.3 \mathrm{fm}$, employing three values of the lattice spacing and two values of the pion mass constructed from sea quarks only.

The XXVIII International Symposium on Lattice Field Theory, Lattice2010

June 14-19, 2010

Villasimius, Italy

\footnotetext{
*Speaker.

${ }^{\dagger}$ Supported by Ministry of Science and Higher Education grant nr. N N202 237437. The computer time for this project was made available to us by the Leibniz Rechenzentrum in Munich, Poznań Supercomputing and Networking Center in Poznań, GENCI-CINES grant 2010-052271 and CCIN2P3 in Lyon. We thank these computer centers and their staff for all technical advice and help.
} 


\begin{tabular}{|c|c|c|c|c|c|c|}
\hline Lattice size & $L[\mathrm{fm}]$ & $\beta$ & $a[\mathrm{fm}]$ & $a \mu$ & $m_{\pi}[\mathrm{MeV}]$ & \#conf \\
\hline \hline $16^{3} \times 32$ & 1.3 & 3.9 & 0.079 & 0.004 & 300 & 544 \\
$20^{3} \times 40$ & 1.3 & 4.05 & 0.063 & 0.003 & 300 & 300 \\
$24^{3} \times 48$ & 1.3 & 4.2 & 0.051 & 0.002 & 300 & 401 \\
$16^{3} \times 32$ & 1.3 & 3.9 & 0.079 & 0.0074 & 450 & 260 \\
$20^{3} \times 40$ & 1.3 & 4.05 & 0.063 & 0.006 & 450 & 299 \\
$24^{3} \times 48$ & 1.3 & 4.2 & 0.051 & 0.005 & 450 & 137 \\
$20^{3} \times 40$ & 1.6 & 3.9 & 0.079 & 0.004 & 300 & 239 \\
$24^{3} \times 48$ & 1.9 & 3.9 & 0.079 & 0.004 & 300 & 435 \\
\hline
\end{tabular}

Table 1: We give the simulation parameters and indicate the values of $L, a$ and $m_{\pi}$ to provide estimates for the physical situation.

\section{Introduction}

Mixed action simulations with overlap fermions are considered to be a cost-effective alternative to fully dynamical overlap simulations. They allow to profit from the good chiral properties of overlap fermions in the valence sector, while in the sea sector a cheaper fermion discretization is used to keep the simulation time to a tolerable level.

We have used several ensembles of gauge field configurations generated by the European Twisted Mass Collaboration (ETMC) to investigate the particular setup of overlap valence quarks on a maximally twisted mass sea. Our aim was to explore the potential of this approach and study the continuum limit behaviour of this mixed action setup. For earlier results regarding this setup, we refer to $[1,2,3]$.

\section{Simulation setup}

We use ETMC $N_{f}=2$ dynamical maximally twisted mass (MTM) gauge field configurations and we refer to refs. $[4,5]$ for the details concerning their generation. The parameter values are gathered in Tab. 1. We focus mainly on small-volume ensembles with $L \approx 1.3 \mathrm{fm}$, with pion masses corresponding to about 300 and $450 \mathrm{MeV}$, but we also consider larger volumes to investigate the size of finite volume effects in the case of the coarsest lattice spacing.

In the valence sector, we use overlap fermions [6], defined by:

$$
\hat{D}_{\text {overlap }}(0)=\frac{1}{a}\left(1-A\left(A^{\dagger} A\right)^{-1 / 2}\right) \text {. }
$$

with the kernel operator $A=1+s-a \hat{D}_{\text {Wilson }}(0)$, where $s$ is a parameter which satisfies $|s|<1$ and can be used to optimize locality properties and the Wilson-Dirac operator is defined by:

$$
\hat{D}_{\text {Wilson }}(m)=\frac{1}{2}\left(\gamma_{\mu}\left(\nabla_{\mu}^{*}+\nabla_{\mu}\right)-\operatorname{ar} \nabla_{\mu}^{*} \nabla_{\mu}\right)+m,
$$

where $m$ is the bare Wilson quark mass and $\nabla_{\mu}, \nabla_{\mu}^{*}$ are the forward and backward covariant derivatives, respectively. The massive overlap Dirac operator is given by:

$$
\hat{D}_{\text {overlap }}\left(m_{q}\right)=\left(1+s-\frac{a m_{q}}{2}\right) \hat{D}_{\text {overlap }}(0)+m_{q},
$$



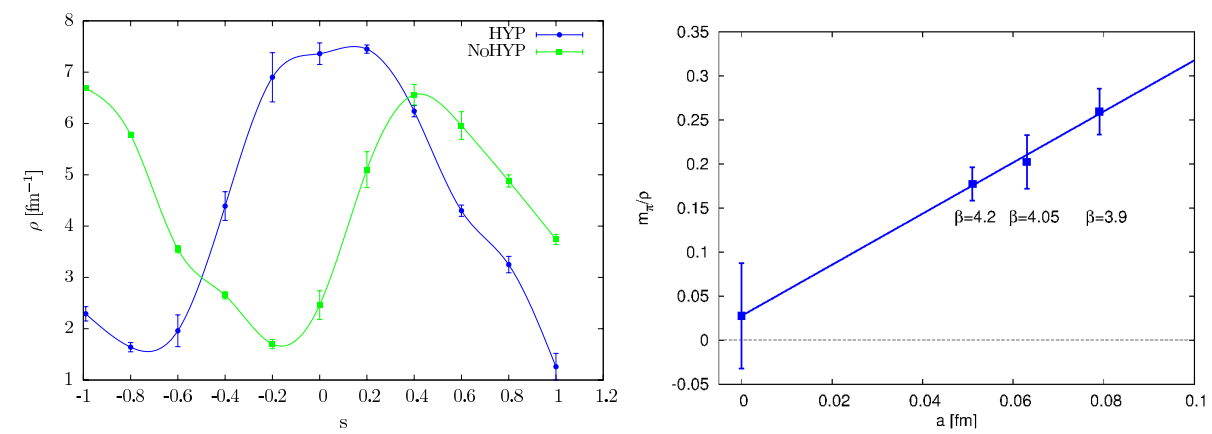

Figure 1: (left) Decay rate of the overlap operator. (right) Continuum limit scaling of the ratio $m_{\pi} / \rho$.

where $m_{q}$ is the bare overlap quark mass.

For each ensemble, following ref. [7] we use the SUMR solver with adaptive precision and multi mass capability to compute the propagators for a wide range of valence quark masses $m_{q}$, from quark mass matched to the unitary one to around the mass of the physical strange quark. Before applying the overlap operator, we perform one iteration of HYP smearing [8] on the gauge field configurations.

\section{Locality of the overlap operator}

In order to achieve the best locality properties of the overlap operator, the parameter $s$ can be tuned. It has been shown [9] that the overlap operator is local for a wide range of simulation parameter values, i.e. the norm of the overlap operator falls off exponentially $\left\|D_{\text {overlap }}\right\|_{\max }(d) \propto$ $e^{-\rho d}$, where $d$ is the taxi-driver distance between lattice points and $\|\cdot\|_{\max }$ is the same norm as used in ref. [9]. We have computed the decay rate $\rho$ for several values of the parameter $s$, both for the overlap operator constructed on HYP smeared and on original, unsmeared configurations.

We find (the left panel of Fig. 1) that in the HYP-smeared case the best locality is observed in the neighbourhood of the free-field optimal value $s=0$. Following this observation, we set $s=0$ in our computations. We also investigate the continuum limit of the ratio $m_{\pi} / \rho$. At finite lattice spacing the condition $m_{\pi}<\rho$ must hold [10] in order that the interaction can be considered local from the point of view of the considered particle. For the pion, the ratio $m_{\pi} / \rho$ (right panel of Fig. 1 ) is well below 1 and thus locality is guaranteed. Moreover, the continuum limit value of $m_{\pi} / \rho$ is consistent with zero, which is related to the fact that $m_{\pi}$ had been fixed to $m_{\pi} \approx 300 \mathrm{MeV}$ and has a non-zero value in the continuum, while $1 / \rho$ vanishes for $a=0$.

\section{Continuum limit scaling test of the pion decay constant}

To minimize the unitarity violations present in the mixed action setup, a procedure of matching of the quark masses has to be performed. In general, there is a large freedom to choose a matching condition. In this proceeding, we will discuss a particular matching condition which will illustrate the role of chiral zero modes of the overlap operator especially. Our condition consists of matching 

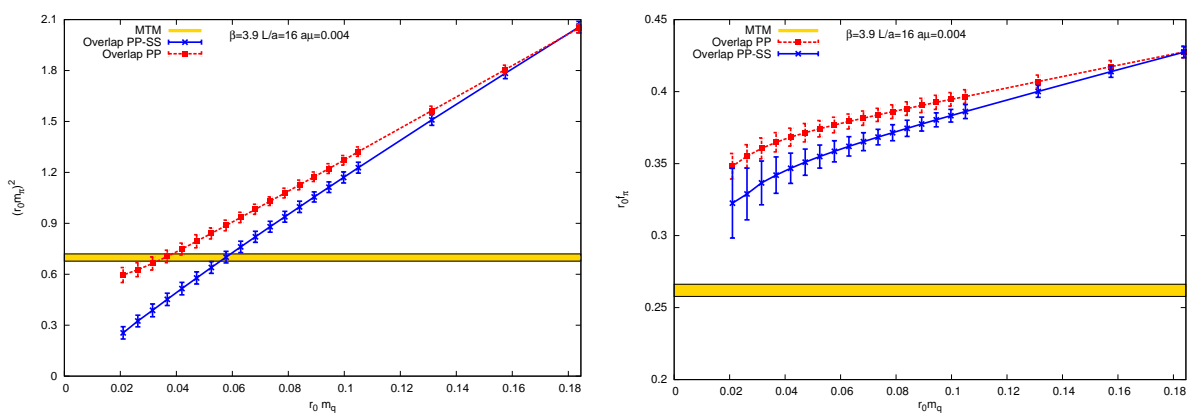

Figure 2: The quark mass dependence of the pion mass (left) and the pion decay constant (right). Ensemble: $\beta=3.9, L / a=16, m_{\pi} \approx 300 \mathrm{MeV}$.
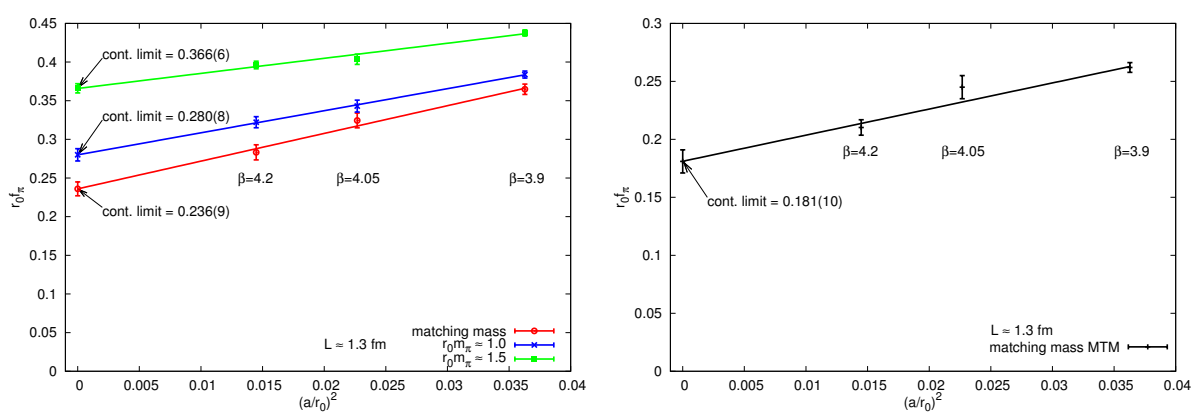

Figure 3: The continuum limit scaling of the pion decay constant (extracted from the PP correlator) overlap pion at the matching mass and two other reference values of $r_{0} m_{\pi}$ (left) and MTM pion (right).

the value of the charged pion mass ${ }^{1}$, i.e. to enforce that the pion built from two valence quarks has a mass closest to the one built from two sea quarks. The procedure of matching is illustrated in the left panel of Fig. 2, which depicts the quark mass dependence of the pion mass. We extract the pion mass from two correlators - the pseudoscalar one (PP) and the difference of the pseudoscalar and the scalar ones (PP-SS). Taking the latter, one can avoid any effects from the chiral zero modes of the overlap operator, since the zero modes couple equally to the PP and SS correlators. Thus, we calculate two matching quark masses for each ensemble.

It is clear that the effects of zero modes are non-negligible for this ensemble and the two definitions of the matching mass lead to different values of the matching quark masses. Hence, we will use both definitions and analyze the effects at both matching quark masses.

The right panel of Fig. 2 shows the quark mass dependence of the pion decay constant $f_{\pi}$, where this observable has been extracted again from both the PP and the PP-SS correlator. The two curves tend to each other for large values of the quark mass, where the effects of the zero modes are small, but for small quark masses (including the matching mass) these effects are rather large.

In the following, we concentrate on the continuum limit scaling test of the pion decay constant.

\footnotetext{
${ }^{1}$ In the twisted mass formalism, the neutral and charged pions have unequal masses, which is due to $\mathscr{O}\left(a^{2}\right)$ isospin symmetry breaking. We choose to match the charged pion mass, because of its smaller statistical uncertainty and smaller discretization errors.
} 

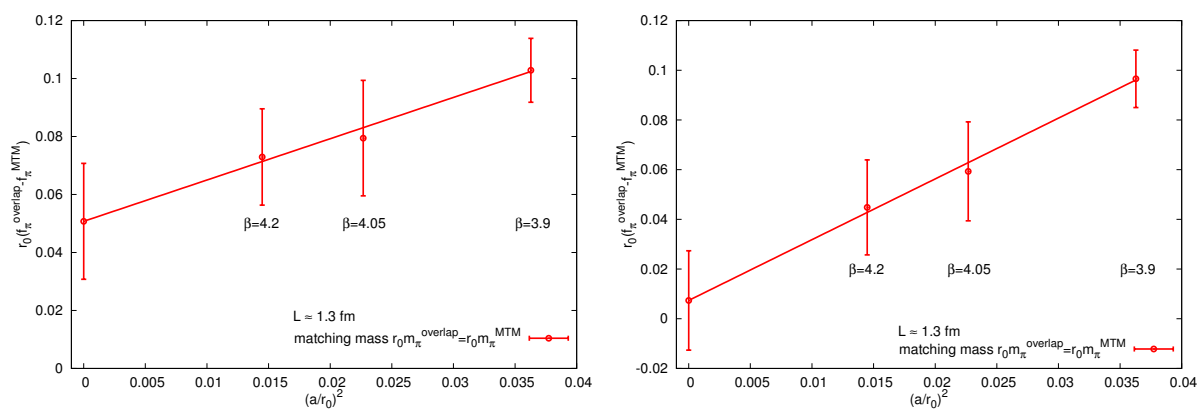

Figure 4: The continuum limit scaling of the difference $r_{0}\left(f_{\pi}^{\text {overlap }}-f_{\pi}^{M T M}\right)$, where $f_{\pi}^{\text {overlap }}$ has been extracted from the PP (left) and the PP-SS correlator (right).
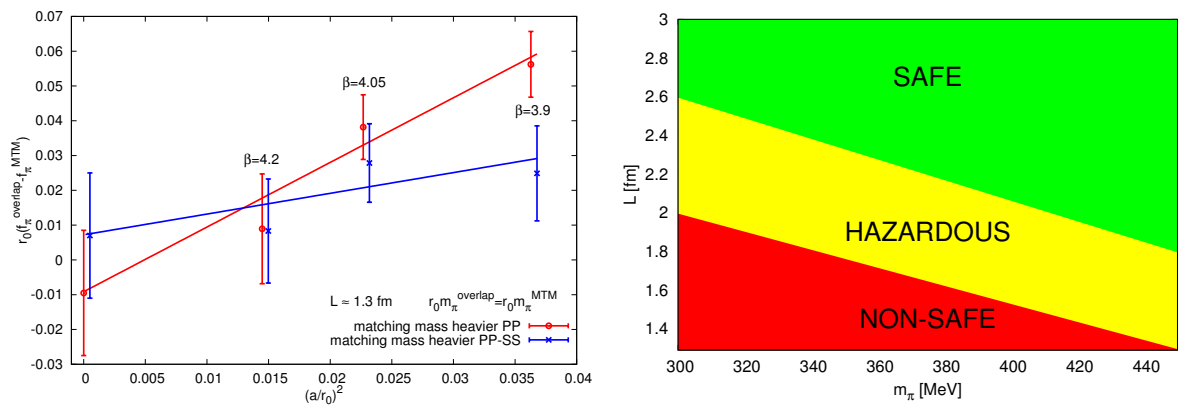

Figure 5: (left) The continuum limit scaling of the difference $r_{0}\left(f_{\pi}^{\text {overlap }}-f_{\pi}^{M T M}\right)$ in the case of a heavier sea quark mass $\left(m_{\pi} \approx 450 \mathrm{MeV}\right)$. (right) The safe, hazardous and non-safe regions of parameter space with respect to the zero modes effects.

We will perform it at three values of the pion mass, corresponding to $r_{0} m_{\pi} \approx 0.85,1.0,1.5$, where the smallest value corresponds to the matching mass. Fig. 3 shows the scaling of $f_{\pi}$ (extracted from the PP correlator) for overlap valence quarks (left) and MTM valence quarks (unitary setup; right). Clearly, both discretizations show $\mathscr{O}\left(a^{2}\right)$ leading discretization effects.

However, when employing the matching condition discussed above, the continuum limit of $f_{\pi}$ in the mixed action setup is different from the unitary one, if $f_{\pi}$ is extracted from the PP correlator (left panel of Fig. 4) at the values of the lattice spacing used here. This can be attributed to the effects of the zero modes, since the continuum limit value of $f_{\pi}$ extracted from the PP-SS correlator agrees nicely with the unitary value (Fig. 4, right). We note that the seemingly inconsistent continuum limits originate from the particular matching condition used here. Employing alternative matching conditions can substantially alter this effect $[11,12]$.

The hypothesis about the role of the zero modes can be confirmed by performing an analogous scaling test for a heavier value of the sea quark mass (corresponding to a pion mass of about 450 $\mathrm{MeV}$ ). In this case, the zero modes effects (which enter the PP correlator as $1 / m_{q}^{2}$ and $1 / m_{q}$ effects) should be reduced. Indeed, the left panel of Fig. 5 shows that the mixed action and unitary values of $f_{\pi}$ agree in the continuum limit, both in the PP and the PP-SS case. The effects of the zero modes are still visible, since $f_{\pi}$ extracted from PP and from PP-SS slightly differ, but these effects 

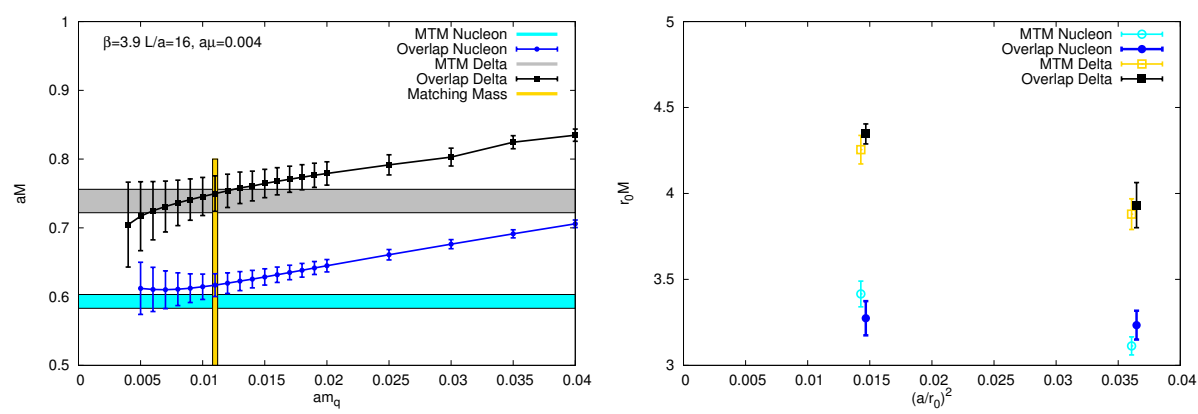

Figure 6: (left) The quark mass dependence of the nucleon and delta mass. Ensemble: $\beta=3.9, L / a=16$, $a \mu=0.004$. (right) The unitary (MTM) and mixed action (overlap) light baryon masses vs. $a^{2}$.

are strongly suppressed with respect to the $m_{\pi} \approx 300 \mathrm{MeV}$ case. Hence, when using a mixed action setup with overlap valence quarks, special attention has to be paid to the effects of the zero modes.

The investigation of finite volume effects at the coarsest lattice spacing, together with the analysis of the sea quark mass dependence at small volume, allowed us to find the range of parameter values that ensure that one is safe against the effects of zero modes. This is summarized in the right panel of Fig. 5. In terms of $m_{\pi} L$ the safe region corresponds to $m_{\pi} L>4$ and the non-safe one to $m_{\pi} L<3$. For the details of this analysis, we refer to an upcoming publication [11].

\section{Light baryon masses}

In order to investigate the effects of the mixed action setup in other observables, we have computed the light baryon masses in the mixed action and the unitary setup. We have used smearedsmeared correlators with the same setup as in [13]. The quark mass dependence of the nucleon and delta masses for one ensemble are depicted in Fig. 6 (left). At the matching mass (indicated by the vertical bar), the masses in both setups are compatible with each other, within statistical error. The situation is similar at a smaller lattice spacing, which is shown in the right panel of Fig. 6. This indicates that the zero mode effects are much smaller in the baryon sector, at least in the case of light baryon masses. For more details about this analysis, we refer to an upcoming publication [12].

\section{Unitarity violations in the scalar correlator}

The final aspect that we shortly discuss are the unitarity violations in the scalar correlator, present in the mixed action setup. As we have already stated, they can be minimized by matching the quark masses, but even at the matching mass they can not be entirely eliminated. One effect of this kind regards the scalar correlation function, which can obtain an unphysical negative contribution from one kind of diagrams. This effect in the mixed action setup has been analyzed within the framework of Partially Quenched Chiral Perturbation Theory in ref. [14]. At large time $t$, the dominant contribution to the scalar correlation function is:

$$
C_{S S}(t) \stackrel{t \rightarrow \infty}{=}-\frac{B_{0}^{2}}{2 L^{3}} \frac{e^{-2 M_{V V} t}}{M_{V V}^{3}} \gamma_{S S} a^{2} t .
$$



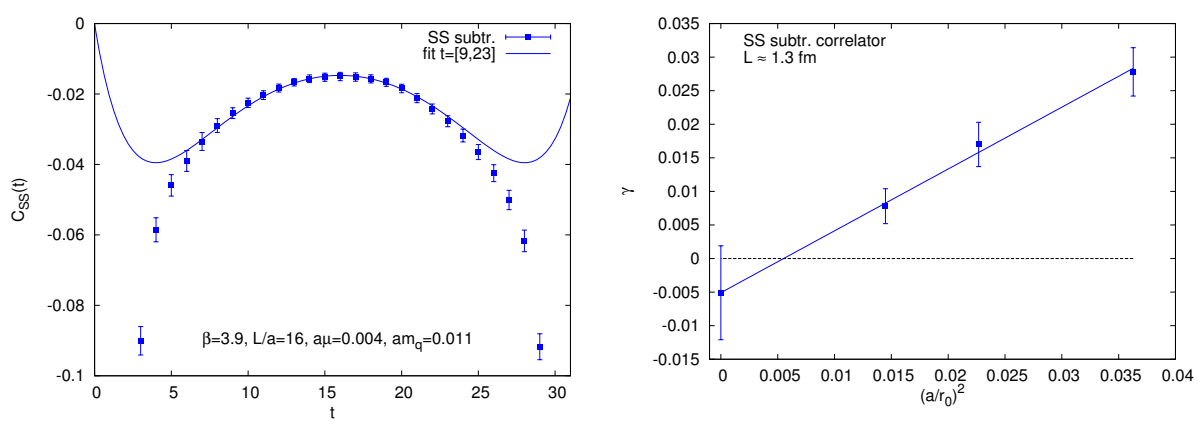

Figure 7: (left) The SS subtr. correlation function at the matching mass . (right) Continuum limit scaling of the fitting parameter $\gamma$.

If we define $\gamma \equiv \frac{B_{0}^{2} \gamma_{S S}}{2\left(M_{V V} L\right)^{3}} a^{2}$ and take periodic boundary conditions in time, we obtain: $C_{S S}(t) \stackrel{t \rightarrow \infty}{=}$ $-\gamma\left(t e^{-2 M_{V V} t}+(T-t) e^{-2 M_{V V}(T-t)}\right)$. In order to analyze this formula, we use the SS correlator with explicitly subtracted zero modes - a field theoretically not clean procedure which we use here only to have some estimate of the unitarity violation effect. We then extract $\gamma$ for each light-quark, small-volume ensemble, by fitting formula (6.1) to the averaged SS correlator. An example of such fit is depicted in Fig. 7 (left). The right panel of this figure shows the extrapolation of $\gamma$ to the continuum limit. The linear dependence in $a^{2}$ and the value consistent with zero for $a=0$ is compatible with the hypothesis that the unitarity violations in the mixed setup are $\mathscr{O}\left(a^{2}\right)$ effects and can be described by formula (6.1). For more details about this test, we again refer to [12].

\section{References}

[1] O. Bär, K. Jansen, S. Schaefer, L. Scorzato, A. Shindler, PoSLAT2006, 199 [hep-1at/ 0609039 ].

[2] N. Garron, L. Scorzato, PoSLAT2007, 083 [0710.1582 (hep-lat)].

[3] K. Cichy, G. Herdoiza, K. Jansen, Acta Phys.Pol.B Proc.Suppl. 2, 497 (2009) [0910 . 0816 (hep-lat)].

[4] P. Boucaud et al., Phys. Lett. B650 (2007), 304; [hep-lat/ 0701012 ].

[5] P. Boucaud et al., Comput. Phys. Commun. 179 (2008) 695-715 [0803.0224 (hep-lat)].

[6] H. Neuberger, Phys. Lett. B 417, 141 (1998); [hep-lat/9707022].

[7] T. Chiarappa et al., hep-lat/0609023.

[8] A. Hasenfratz, F. Knechtli, Phys.Rev. D64, 034504 (2001); [hep-lat/ 0103029 ].

[9] P. Hernandez, K. Jansen, M. Lüscher, Nucl.Phys. B552 (1999) 363-378 [hep-lat/9808010].

[10] F. Niedermayer, Nucl. Phys. B (Proc. Suppl.) 73 (1999), 105 [hep-lat/9810026].

[11] K. Cichy, G. Herdoiza, K. Jansen (2010), in preparation.

[12] K. Cichy, V. Drach, E. Garcia Ramos, G. Herdoiza, K. Jansen (2010), in preparation.

[13] C. Alexandrou et al., Phys.Rev. D 78, 014509 (2008); [0803.3190 (hep-lat) ].

[14] M. Golterman, T. Izubuchi, Y. Shamir, Phys.Rev. D71, 114508 (2005); [hep-lat / 0504013 ]. 